\title{
Structure and Dynamics of the Crystalline Stable Phase of 2-Chlorothiophene
}

\author{
Michela Romanini, ${ }^{\dagger}$ Philippe Negrier, ${ }^{\ddagger}$ Maria Barrio, ${ }^{\dagger}$ Denise Mondieig, ${ }^{\ddagger}$ Pablo Serra, ${ }^{\S}$ \\ Mariano J. Zuriaga, ${ }^{\S}$ Roberto Macovez, ${ }^{\dagger}$ and Josep-Lluís Tamarit ${ }^{*}{ }^{\dagger}$ (i) \\ ${ }^{\dagger}$ Grup de Caracterització de Materials, Departament de Física and Barcelona Research Center in Multiscale Science and Engineering, \\ Universitat Politècnica de Catalunya, EEBE, Campus Diagonal-Besòs, Av. Eduard Maristany 10-14, 08019 Barcelona, Catalonia, \\ Spain \\ ${ }^{\ddagger}$ LOMA, UMR 5798, CNRS, Université de Bordeaux, F-33400 Talence, France \\ ${ }^{\S}$ Facultad de Matemática, Astronomía, Física y Computación, Universidad Nacional de Córdoba and IFEG-CONICET, Ciudad \\ Universitaria, X5016LAE Córdoba, Argentina
}

\section{Supporting Information}

ABSTRACT: The dynamics of a simple and rigid molecule (2-chlorothiophene) has been studied by means of broadband dielectric spectroscopy (BDS) within the low-temperature stable crystalline phase, whose structure has been determined by means of powder X-ray diffraction (PXRD) measurements. The triclinic $P 1$ structure consists of two molecules per asymmetric unit $\left(Z^{\prime}=2\right)$ with site occupancies of 80:20 and 60:40 for each molecule of the asymmetric unit. Such a statistical intrinsic disorder is associated with two dynamical relaxation processes corresponding respectively to out-of-plane relaxations around the $\mathrm{C}-$ $\mathrm{Cl}$ 2-fold molecular axis and in-plane reorientational motions as proposed previously (Fujiromi and Oguni, J. Phys. Chem. Solids 1993, 54, 437-612). The PXRD and BDS measurements allow rationalizing published nuclear quadrupole resonance data. We show that only the concurrent use of different experimental techniques provides the answer to a complicated case of orientational molecular dynamics in the solid state.

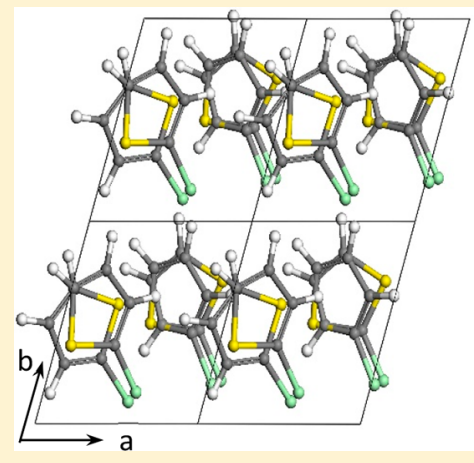

\section{INTRODUCTION}

Structural glasses are obtained by cooling or pressurizing liquids, which are ergodic disordered systems with both translational and orientational disorder. ${ }^{1-3}$ The dynamics of the system slows down fast enough to avoid the transition to a more ordered state, generally the fully ordered crystalline state. When the ergodic state is translationally ordered but orientationally disordered (as in a plastic crystal or a rotator phase), the reorientational motions of the molecules also can be frozen in the same way, giving rise also to a glassy state called orientational glass and exhibiting the same dynamic features and thermodynamic anomalies. ${ }^{4-12}$ As far as dynamic processes are concerned, in addition to the omnipresent cooperative $\alpha$ relaxation (ascribed in structural glasses to their viscous flow), secondary relaxations with shorter relaxation times appear, which may have different origins. ${ }^{6,13-19}$ Among them, the Johari-Goldstein $\beta$ relaxation process exhibited even by rigid molecules is quite common and can be interpreted on the basis of the energy-landscape picture as jumps between the basins $^{20,21}$ within a metabasin and generally follows the predictions of the coupling model. ${ }^{22-24}$ The existence of dynamic processes has been made evident even for highly ordered systems such as translationally ordered phases with an intrinsic statistical disorder involving only the site occupancy of one or a few atoms of the (rigid or flexible) molecular entities. $^{9-12,25-29}$ In these cases, the distinct site occupancy probabilities (called fractional occupancies) reflect the existence of perfectly defined, discrete allowed angular orientations of the molecules that undergo reorientational jumps between the allowed orientations, in contrast to the undefined and hardly quantifiable dynamics of translationally and orientationally disordered phases such as the liquid state. The study of these minimally disordered crystalline systems with few and quantifiable disordered configurations can allow the precise identification of the different dynamic processes observed experimentally, as well as on the required minimal disorder for the emergence of the so-called universal thermal anomalies of the glass state.

Here we report on the low-temperature crystalline phase of 2-chlorothiophene $\left(\mathrm{C}_{4} \mathrm{H}_{3} \mathrm{ClS}\right)$, a derivative of the fivemembered sulfur heterocyclic thiophene $\left(\mathrm{C}_{4} \mathrm{H}_{4} \mathrm{~S}\right)$ molecule. Thiophene is a very simple and rigid molecule that displays a rich polymorphism, with five stable and three metastable crystalline phases, for which large amplitude in-plane molecular reorientations have been reported. ${ }^{30,31}$ The easiness of the reorientational motions was attributed to the molecular shape (similar to a short cylinder) and to the van der Waals interactions, both allowing rotations between distinct discrete

Received: July 4, 2019

Revised: September 13, 2019

Published: September 23, 2019 
orientations around the quasi 5-fold axis perpendicular to the molecular plane within the herringbone structure of the different polymorphs. ${ }^{30}$ This "simple" system is very sensitive to subtle changes of the intermolecular interactions, as proven by the reported effect of isotopic substitution of $\mathrm{H}$ by deuterium: in the low-temperature crystal phase of $\mathrm{C}_{4} \mathrm{D}_{4} \mathrm{~S}$ (phase $\mathrm{V}$, stable below $112 \mathrm{~K}$ ), the molecules are perfectly ordered, unlike the low-temperature phase of $\mathrm{C}_{4} \mathrm{H}_{4} \mathrm{~S}$ which is disordered with a reported glassy transition temperature at 42 $\mathrm{K} .{ }^{32}$ It was speculated that the in-plane reorientational motion in the hydrogenated compound is prevented in the deuterated compound due to the larger molecular mass and thus a larger moment of inertia. Solid methane $\left(\mathrm{CH}_{4}\right)$ is another example of a system where subtle changes in the disorder are caused by isotopic effects. ${ }^{33}$

The 2-chlorothiophene derivative was first studied by means of adiabatic calorimetry within the range of 13 and $300 \mathrm{~K}^{34}$ and few years later by nuclear quadrupole resonance (NQR) from $200 \mathrm{~K}$ down to $77 \mathrm{~K}^{35}$ Calorimetric measurements revealed two thermal anomalies in the crystalline phase (melting temperature of $201.3 \mathrm{~K}$ ) at 164 and $186 \mathrm{~K}$. From the calorimetric measurements, the authors proposed the existence of two different kinds of motions, namely, 5-fold rotations (of $2 \pi / 5$ angular amplitude) around an axis perpendicular to the molecular plane, and 2-fold out-of-plane $(\pi)$ rotations about the $\mathrm{C}-\mathrm{Cl}$ molecular axis. In terms of the crystalline structure detectable in XRD measurements, the occurrence of in-plane rotations would imply fractional occupancy probabilities for both the chlorine and sulfur atoms, while the out-of-plane rotations would lead only to a disorder of the sulfur atom within the asymmetric unit cell (assuming that such a rotation keeps the molecular plane invariant). The NQR study pointed out the existence of two broad $\left(100 \mathrm{kHz}\right.$ full width at half-maximum) ${ }^{35} \mathrm{Cl}$ signals with almost identical temperature variation in the measured temperature range. Although this was not acknowledged by the authors of the NQR study, the detection of two distinct ${ }^{35} \mathrm{Cl} \mathrm{NQR}$ signals actually reveals the existence of two independent chlorine atoms within the asymmetric unit of the crystal. The temperature variation of the spin-lattice relaxation time $\left(T_{1}\right)$ was accounted for assuming two dynamic processes, namely, the typical libration modes active also at low-temperature $\left(T_{1} \propto T^{n}\right.$, with $\left.n \approx 3.1\right)$ and an activated process $\left(T_{1} \propto \mathrm{e}^{-\mathrm{E} / R T}\right)$ with an activation energy of $53 \mathrm{~kJ} \cdot \mathrm{mol}^{-1}$. The lack of information about the crystal structure prevented a rationalization of the physical origin of the NQR data as well as a compatibility check to discard or support the existence of the proposed molecular motions.

In this work, we determine experimentally the crystal structure and dielectric relaxation dynamics of the lowtemperature phase of 2-chlorothiophene and reanalyze the NQR data with these new pieces of evidence. We demonstrate that the out-of-plane rotations are accompanied by a small change of the molecular plane, in particular, by a change of the orientation of the $\mathrm{C}-\mathrm{Cl}$ bond. The in-plane rotations proposed early by Fujimori and Oguni ${ }^{34}$ can only appear as short-lived molecular fluctuations undetectable by X-ray diffraction; they must therefore involve at least one nonequilibrium configuration as it was recently found in some haloethane compounds. ${ }^{25,36}$ These results allow a microscopic identification of both the primary and secondary dielectric relaxation processes that we observe experimentally.

\section{MATERIALS AND METHODS}

2-Chloro-thiophene $\left(\mathrm{C}_{4} \mathrm{H}_{3} \mathrm{ClS}, M_{\mathrm{w}}=118.58 \mathrm{~g} / \mathrm{mol}\right)$ was purchased from Sigma-Aldrich with a purity higher than $96 \%$ and used as provided.

Differential scanning calorimetry (DSC) experiments were carried out in both high-pressure stainless-steel and aluminum pans under nitrogen atmosphere, by means of a DSC-7 calorimeter from PerkinElmer. Measurements were performed in the temperature range from $170 \mathrm{~K}$ to room temperature, with a heating/cooling rate of $2 \mathrm{~K} / \mathrm{min}$ and masses around $10 \mathrm{mg}$.

High resolution X-ray diffraction patterns were recorded at room temperature with a cylindrical position-sensitive detector (CPS120) from INEL (France) using monochromatic $\mathrm{Cu}-\mathrm{K} \alpha_{1}(\lambda=1.54056 \AA)$ radiation. The generator power was set to $40 \mathrm{kV}$ and $25 \mathrm{~mA}$. X-ray patterns on a liquid sample sealed in a $0.3 \mathrm{~mm}$ diameter Lindemann capillary, which were rotated around their axes during data collection to improve averaging of the crystallites, were obtained as a function of temperature (from $90 \mathrm{~K}$ to the melting point) by means of a 600 series cryostream cooler from Oxford Cryosystems.

External calibration was performed by means of cubic phase $\mathrm{Na}_{2} \mathrm{Ca}_{3} \mathrm{Al}_{2} \mathrm{~F}_{4}$ mixed with silver behenate. The peak positions were determined by pseudo-Voigt fittings.

The acquisition times were at least $7 \mathrm{~h}$ for the structural determinations at low-temperature and $30 \mathrm{~min}$ for a pattern matching procedure to determine the variation of the lattice as a function of temperature. Indexing of the X-ray powder diffraction patterns, structure solutions, and Pawley and Rietveld refinements were performed using the Materials Studio Program. ${ }^{37}$

Broadband dielectric spectroscopy (BDS) measurements were carried out using a Novocontrol Alpha analyzer. The liquid sample was placed in a stainless steel parallel-plate capacitor specially designed for the analysis of liquid samples, with the two electrodes kept at a fixed distance by means of silica spacers of $50 \mu \mathrm{m}$ diameter. Temperature control was achieved with a nitrogen-gas flow cryostat (Quatro) with an error not higher than $0.3 \mathrm{~K}$. Measurements were done in the frequency $(\nu)$ range between $10^{-2}$ and $10^{6} \mathrm{~Hz}$.

Relaxation time values were obtained by fitting the dielectric loss spectra with a power law for the conductivity contribution and a superposition of Cole-Cole (CC) functions for the relaxation peaks. The analytical expression of the CC function is

$$
\varepsilon^{*}(\omega)=\varepsilon^{\prime}-i \varepsilon^{\prime \prime}=\varepsilon_{\infty}+\frac{\Delta \varepsilon}{1+(i \omega \tau)^{c}}
$$

where $\varepsilon_{\infty}$ is the permittivity in the high frequency limit, $\Delta \varepsilon$ is the dielectric strength, $\omega$ is the circular frequency $(\omega=2 \pi \nu), c$ is the width parameter $(0 \leq c<1)$ describing the shape of the loss curves, and $\tau$ is the characteristic relaxation time The fit functions used to model the relaxation processes were actually of the more general Havriliak-Negami type, whose spectral line shape is asymmetric and depends on a second exponent, but the free fits gave a better agreement with the experimental data when such an exponent was equal to 1 , corresponding to the Cole-Cole case.

\section{RESULTS}

DSC measurements (see Figure S1 in Supporting Information) revealed only the occurrence of the melting of the crystalline phase at $200.4 \pm 1.0 \mathrm{~K}$ with a transition enthalpy of $\Delta H_{\mathrm{S} \rightarrow \mathrm{L}}=$ $9.2 \pm 0.5 \mathrm{~kJ} \mathrm{~mol}^{-1}$, virtually the same values as those reported by Fujimori and Oguni $^{34}$ from adiabatic calorimetry $(201.3 \mathrm{~K}$ and $\left.8.966 \mathrm{~kJ} \mathrm{~mol}^{-1}\right)$, who employed an extra-pure compound for their measurements.

The X-ray diffraction patterns of the solid phase were indexed by means of the $\mathrm{X}$-Cell software available in the Powder Indexing of Materials Studio modeling software, using a long-time acquisition pattern measured at $160 \mathrm{~K}$. The indexing procedure yielded a triclinic structure with $Z=2$ according to a reasonable molecular density. The lack of 
systematic absences is compatible with two different space groups, namely, the enantiomorphic $P 1$ and the centrosymmetric $P \overline{1}$. NQR experiments ${ }^{35}$ (see below), and Rietveld refinement provided a compatible solution with the $P 1$ space group with $Z=2$.

A Pawley refinement was performed using the initially determined lattice parameters in space group $P 1$, and they were refined together with a zero-point shift, background, peak-profile (under pseudo-Voigt function), and peak asymmetry parameters.

For structural determination, a rigid-body molecule was built up through the Forcite software (also available in the Materials Studio package). Molecular bond distances and angles were, within the available accuracy, in agreement with previously published data for the molecule in the gas phase obtained from electron diffraction. ${ }^{38}$ With such a rigid-body constraint, two molecules of the asymmetric unit $\left(Z^{\prime}=2\right)$, as inferred from the two signals obtained from the NQR experiments, were put in the cell, and the structure was solved through the Powder Solve module including the March-Dollase function as preferred orientations. ${ }^{39}$

The final Rietveld refinement provided the position and orientation of both molecules in the unit cell.

As for the thiophene case, the results indicate that the two independent molecules display a statistical disorder. Several models were then analyzed. A first model was built up assuming that both molecules were perfectly ordered, a hypothesis that is however in disagreement with the dielectric spectroscopy results and NQR data (see below). A second model was based on two disordered molecules for which the respective $\mathrm{S}$ atoms can occupy two different sites related by a 2 -fold rotation around the $\mathrm{C}-\mathrm{Cl}$ molecular axis (assuming that $\mathrm{Cl}$ atoms occupy the same position). Although this model provided reasonable refinement factors, it did not provide the right solution that should contain a disorder related to the $\mathrm{Cl}$ atom, as discussed in the Introduction.

The best solution compatible with the spectroscopic NQR results was based on two molecules each having a different orientation and different occupancy factors. For one of the molecules (molecule M1) in the asymmetric unit, the occupancy factors at $160 \mathrm{~K}$ turned out to be $60: 40$, whereas for the other one (molecule M2) the ratio is found to be 80:20. It should be especially emphasized that for both molecules both the $\mathrm{S}$ and $\mathrm{Cl}$ atoms (which are largely responsible for the molecular dipole moment) are disordered between two sites, in such a way that the $\mathrm{C}-\mathrm{Cl}$ angle difference between each occupied site for molecule $\mathrm{M} 1$ is around $2.4^{\circ}$, whereas for molecule M2 it is $6^{\circ}$ (see the CIF file for the fractional coordinates at $160 \mathrm{~K})$.

The refinement results based on this model are depicted in Figure 1, in which the calculated profile is shown together with the experimental pattern, their mutual difference, and the Bragg reflections. Final refined parameters are collected in Table S1 of the Supporting Information. Figure 2shows two projections of the $P 1$ triclinic phase of 2 -Cl-thiophene at $160 \mathrm{~K}$ refined structure (along the $c$ and along $b$ planes)

In order to determine the temperature-dependence of the lattice parameters, the liquid sample was cooled relatively fast to $90 \mathrm{~K}$ and $\mathrm{X}$-ray diffraction patterns were subsequently acquired on heating every $10 \mathrm{~K}$ until $160 \mathrm{~K}$ and afterward every 5 or $3 \mathrm{~K}$ until the melting temperature. The results are plotted in Figure 3 for lattice parameters (Figure $3 \mathrm{~A}-\mathrm{F}$ ) as well as for the molar volume (Figure $3 \mathrm{G}$ ). It can be observed

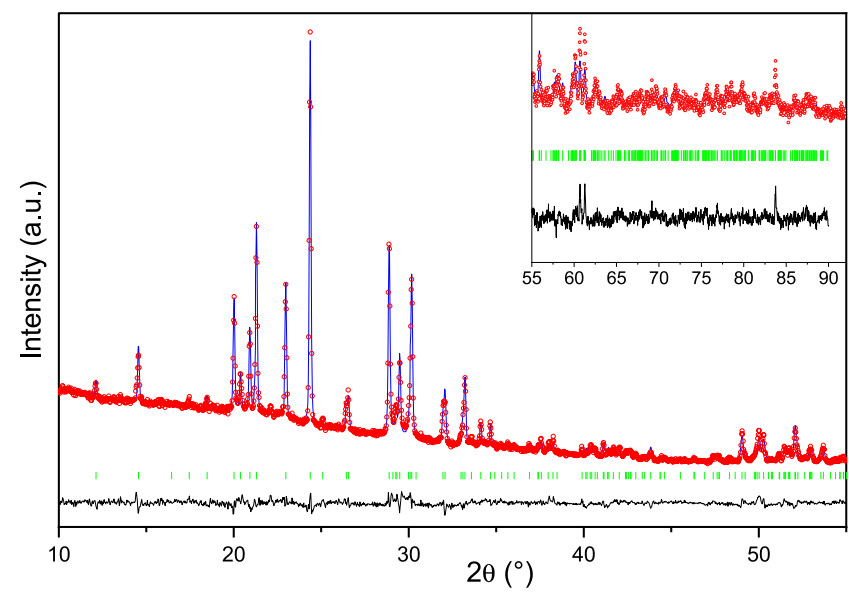

Figure 1. Experimental (red circles) and calculated (blue line) X-ray diffraction patterns along with the difference profile (black line) and Bragg reflections (vertical green sticks) for the final Rietveld refinement of the $P 1$ triclinic phase of 2-Cl-thiophene at $160 \mathrm{~K}$. The inset shows the high angle portion of the pattern (scaled to enhance its visibility).
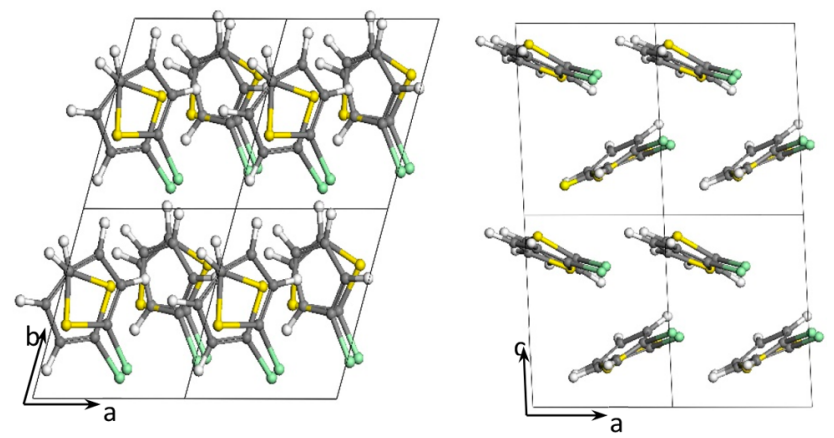

Figure 2. Projection of the refined structure of the $P 1$ triclinic phase of 2-Cl-thiophene at $160 \mathrm{~K}$ along $c$ (left) and along $b$ planes (right). Color of the atoms: $\mathrm{Cl}$ green, $\mathrm{S}$ yellow, $\mathrm{C}$ gray, $\mathrm{H}$ white.

that lattice parameters show a kink at ca. $180 \mathrm{~K}$, without a change in the overall structure.

Figure $3 \mathrm{H}$ displays the difference in $\mathrm{C}-\mathrm{Cl}$ angle between the two possible molecular orientations for both molecules ( 1 and 2) of the asymmetric unit, as determined from Rietveld refinements at each temperature. It can be observed that the angular difference for molecule 2 reveals a noticeable change in the temperature dependence above $190 \mathrm{~K}$, in such a way that the difference between the two molecular orientations increases at high temperature; i.e., the reorientational jumps of the $\mathrm{C}-\mathrm{Cl}$ bond take place between more distant positions.

BDS experiments were carried out in order to verify if the disorder of the triclinic phase of 2-Cl-thiophene found in the structural analysis has a dynamic character. A few representative loss dielectric spectra (imaginary part of the complex relative dielectric permittivity) are shown in Figure 4A. The loss spectra exhibit a conductivity background that is higher at low frequency and increases with increasing temperature, as expected for an organic semiconductor, on top of which a dielectric loss is observed, which increases in intensity and shifts to higher frequency as the temperature is increased. While at low temperature the dielectric loss consists of a single, symmetric loss peak, corresponding to a secondary relaxation (fast process, $\beta$ ), the spectra collected at a higher temperature 

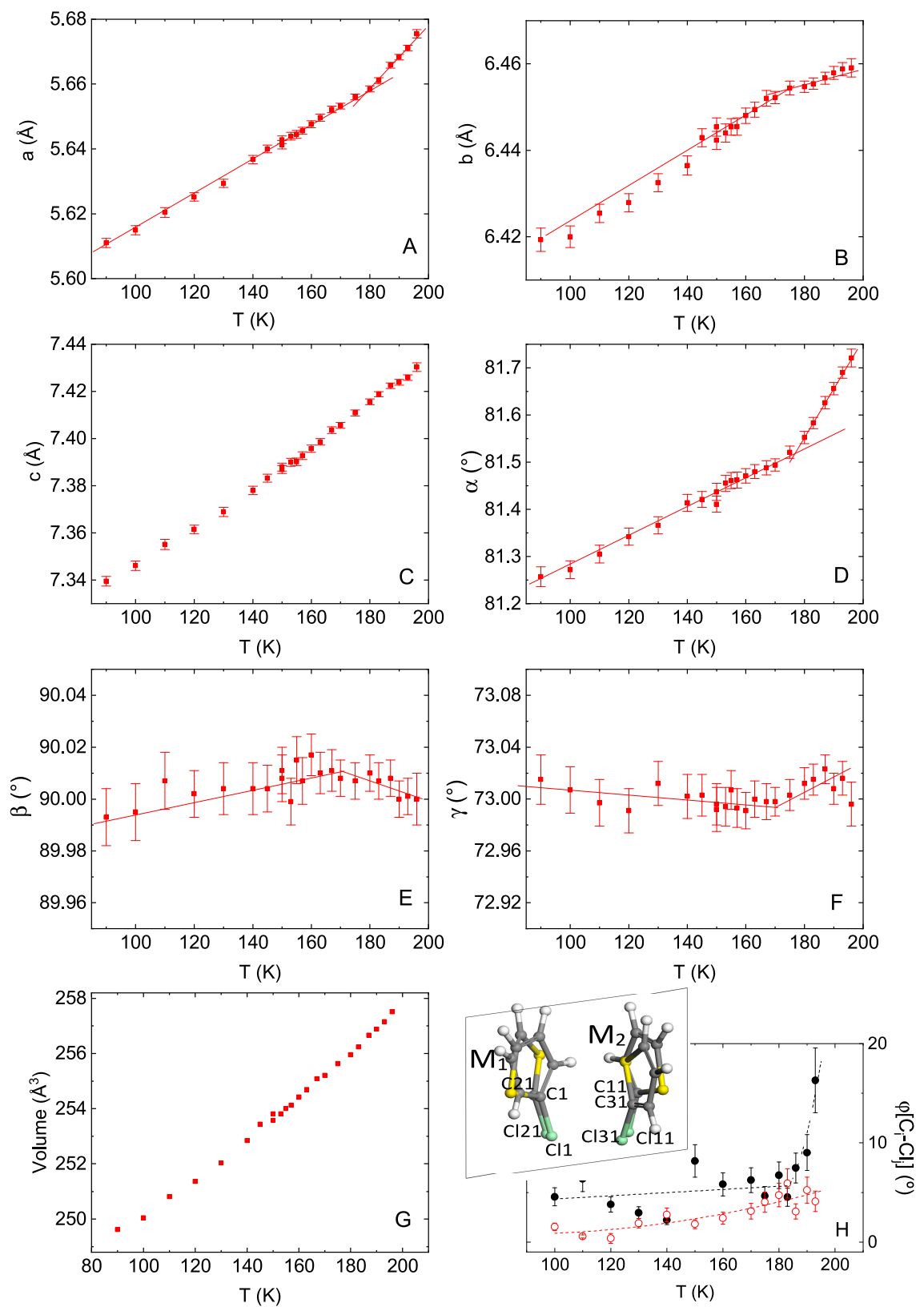

Figure 3. Lattice parameters $(\mathrm{A}-\mathrm{F})$ and molar volume $(\mathrm{G})$ for the stable triclinic $P 1$ phase of 2-Cl-thiophene as a function of temperature, measured on heating after cooling at $90 \mathrm{~K}$. Panel $\mathrm{H}$ depicts the $\mathrm{C}-\mathrm{Cl}$ angle between each occupied site for molecules $\mathrm{M} 1$ (open red circles) and M2 (solid black circles) of the asymmetric unit.

display two distinct components, of which the more intense and slower one (at lower frequency) corresponds to the cooperative primary $\alpha$ relaxation, as detailed below. Each loss component was fitted as the imaginary part of a CC function (eq 1) so as to obtain the characteristic relaxation times for the slow and fast relaxations, $\tau_{\alpha}$ and $\tau_{\beta}$, respectively. Figure $4 \mathrm{~B}$ plots the relaxation times for both processes, which are observed to follow a simply activated Arrhenius behavior $(\tau=$ $\left.\tau_{\mathrm{o}} e^{E / R T}\right)$ with activation energies $E_{\alpha}=82.3 \pm 0.7 \mathrm{~kJ} \mathrm{~mol}^{-1}$ and $E_{\beta}=72.4 \pm 0.7 \mathrm{~kJ} \mathrm{~mol}^{-1}$, respectively. The identification of the slower $\alpha$ process as the primary relaxation follows from the fact that the dynamic glassy transition temperature obtained by our BDS data, defined as the temperature at which the relaxation time $\tau_{\alpha}$ reaches $100 \mathrm{~s}$, is $164 \mathrm{~K}$, which is virtually the same temperature $T_{\mathrm{g} 1}$ found from adiabatic calorimetry. ${ }^{34}$ This would imply that the statistical disorder observed in our PXRD data is dynamic above $164 \mathrm{~K}$ and largely static below this temperature, where the $\alpha$ process is kinetically frozen.

Our assignment of the $\alpha$ relaxation is also confirmed by our analysis of the NQR data, as we discuss in the following. It is interesting to point out that the strength of the $\alpha$ relaxation increases with increasing temperature, especially above approximately $180 \mathrm{~K}$ (see the inset to Figure 4A). This is consistent with our PXRD results, since at this temperature the initial and final molecular orientations after the out-of-plane start differing by larger and larger angles, which entails a larger change in the electric dipole moment of the molecules upon reorientation and thus a larger contribution to the dielectric loss and static dielectric constant.

The interpretation of the secondary $\beta$ relaxation is less straightforward. The fact that the $\alpha$ and $\beta$ relaxations do not merge at high temperature, and the fact that the so-called 

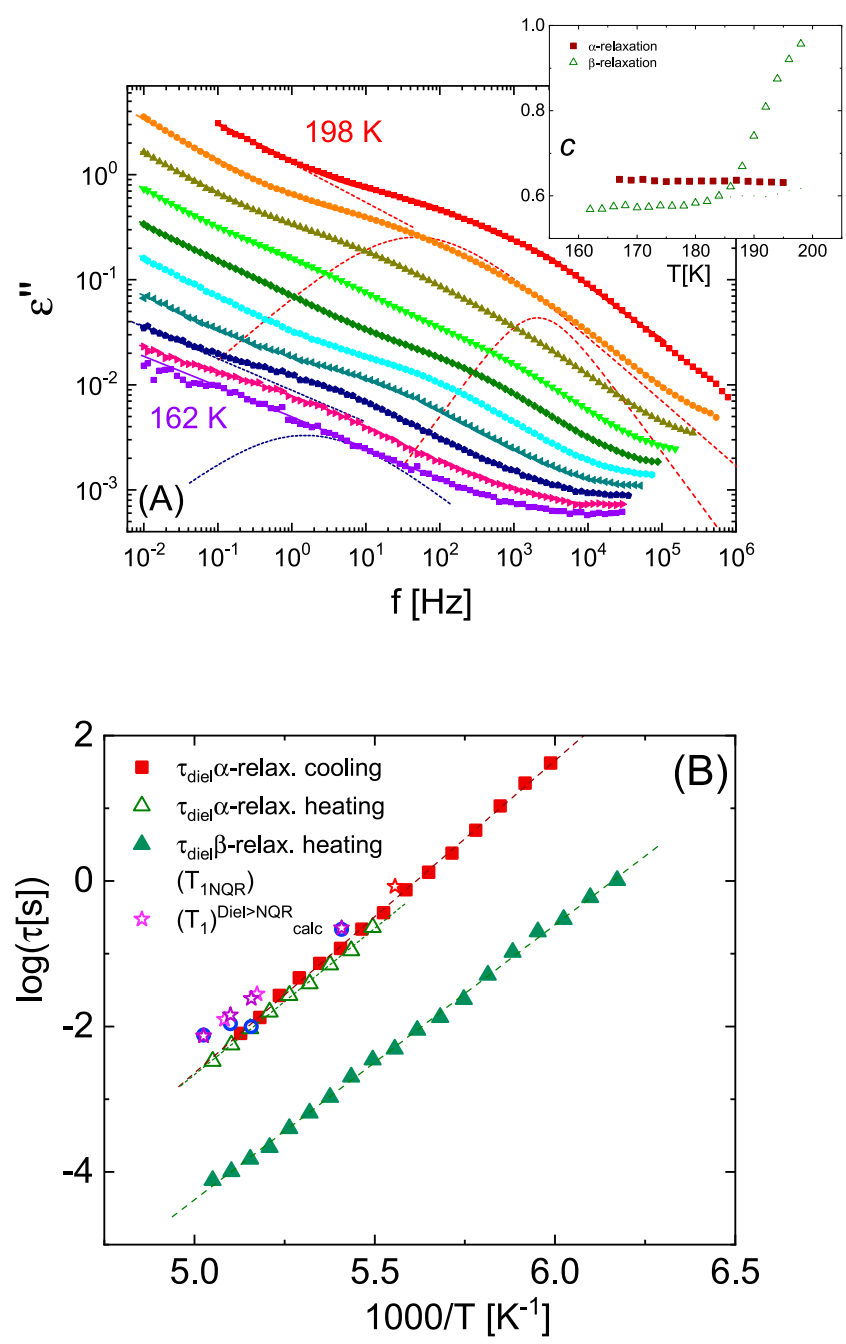

Figure 4. (A) Loss spectra of the stable triclinic phase of 2-Clthiophene measured between 162 and $198 \mathrm{~K}$, every $4 \mathrm{~K}$. Continuous lines are the fits of the spectra at each temperature, and dashed red lines are the fits of conductivity contribution and the slow $(\alpha)$ and fast $(\beta)$ relaxations for the spectrum at $198 \mathrm{~K}$. Blue dashed lines are the conductivity and $\beta$ relaxation contributions at $170 \mathrm{~K}$. Inset: ColeCole width parameter $c(0 \leq c<1)$ describing the shape of the $\alpha$ and $\beta$ loss curves as a function of temperature. (B) Arrhenius plot of the slow relaxation $\tau_{\alpha}$ (filled red squares) and fast $\tau_{\beta}$ (filled green triangles) relaxation times, obtained from the loss dielectric spectra displayed in panel (A). Continuous lines are the fits according to the Arrhenius law. Blue circles are NQR spin-lattice relaxation times minus the lattice vibration contribution $\left(\Delta T_{1}\right)$ and pink stars are the values of $\Delta T_{1}$ generated with eq 4 at the same temperatures.

coupling model ${ }^{22-24}$ does not allow prediction of the relaxation time of the secondary relaxation from the spectral parameters of the primary relaxation, indicate that the $\beta$ relaxation is not of the Johari-Goldstein type. Its lower strength (lower effective dipole moment density) indicates that it might correspond to a small-angle reorientational motion, which could perhaps correspond to small angle ratcheting librations of the molecule, which leaves almost invariant the position of the sulfur atom (no out-of-plane rotation), while changing the position of the chlorine between the two possible crystallographic positions at each site.

\section{DISCUSSION}

As mentioned, our structural analysis has shown the existence of two molecules in the asymmetric unit (and also in the unit cell, $Z=Z^{\prime}=2$ ) with temperature-independent occupancies of 60:40 and 80:20 for molecules labeled as 1 and 2, respectively. For this herringbone structure, the disorder can be interpreted as resulting from an out-of-plane rotation of each molecule around its pseudo-2-fold molecular axis, which is accompanied by a small tilt of the $\mathrm{C}-\mathrm{Cl}$ bond direction (both molecules are not in the same plane before and after the out-of-plane reorientation).

To account for such a disorder, the angle between $\mathrm{C}-\mathrm{Cl}$ bonds between the two occupied sites for each molecule was determined. In other words, the molecular dipole of molecules 1 and 2 of the asymmetric unit can reorient between two different orientations being discernible by the $\varphi_{1}$ and $\varphi_{2}$ represented in Figure $3 \mathrm{H}$. This figure reveals that not only the fractional occupancies are different for molecules 1 and 2, but also slightly the angle between the molecular sites. According to this structural evidence and the emergence of two relaxation processes in the loss dielectric spectra, the two dynamical processes should be ascribed to out-of-plane molecular reorientations between different sites, giving rise to the main slow and cooperative $\alpha$ relaxation, and small angle dipole reorientations due to localized molecular librations ( $\beta$ relaxation).

In order to confirm the origin of the slow $\alpha$ relaxation and provide more insight on that of the fast $\beta$ relaxation, published NQR experiments ${ }^{35}$ were reanalyzed in the light of the structural results from this work.

The temperature dependence of ${ }^{35} \mathrm{Cl} \mathrm{NQR}$ frequency and spin-lattice relaxation time $\left(T_{1}\right)$ were measured in the 77$200 \mathrm{~K}$ range. ${ }^{35}$ The reported NQR spectrum consists in two broad peaks with a full width of about $100 \mathrm{kHz}$ at halfmaximum each, which is an indication of crystal disorder. This broadening arises from random strains, electric fields, and other perturbations from the "defects" in the lattice containing the nuclei whose transitions are studied. ${ }^{40}$ The NQR spectra at $77 \mathrm{~K}$, reproduced in Figure 5A, and the fit with two Gaussian functions show that the area ratio between low and high frequency peaks is 2 . As the unit cell consists of two independent molecules, two peaks of the same intensity are expected a priori, and not a ratio of $2: 1$. This ratio can however

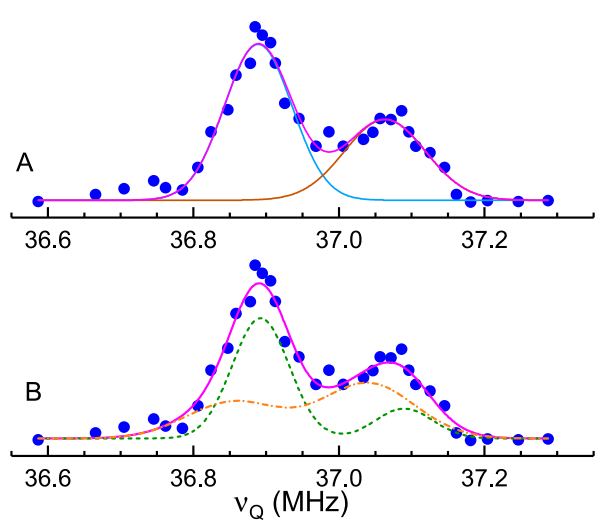

Figure 5. NQR line shape from ref 35. (A) Fit of NQR line with two Gaussians with an intensity ratio 2:1. (B) Fit with the distribution given in eq 2. Dashed line $g(\nu)$ for the chlorine with occupation 60:40, dotted line $g(\nu)$ for chlorine with a fractional occupation 80:20. 
be understood if we consider that the two molecular orientations have different occupation factors. Using statistical methods, it is possible to reproduce these inhomogeneous lineshapes according to the distribution, density, and perturbation fields of individual defects, ${ }^{40,41}$ in ionic crystals ${ }^{42}$ or molecular crystals. ${ }^{43}$ The last model not only reproduces broadening of the NQR lines but also the appearance of satellite lines due to the discreteness of the lattice, ${ }^{43-45}$ and since it was successfully applied in halogenated benzenes and their molecular alloys. ${ }^{46}$

For each quadrupolar nuclei in a nonequivalent site the NQR line width can be accounted by the distribution ${ }^{43}$

$$
g(\nu)=\frac{1}{\sqrt{2 \pi} \sigma}\left(C_{c 1} e^{-\left(\nu-\bar{\nu}-\nu_{c 1,1}\right)^{2} / 2 \sigma^{2}}+C_{c 2} e^{-\left(\nu-\bar{\nu}-\nu_{c 2,1}\right)^{2} / 2 \sigma^{2}}\right)
$$

where $C_{c 1}$ and $C_{c 2}$ are the proportions or occupational factors of molecules with configuration $c 1$ or $c 2$ (the two positions between which the molecule reorientates), $\nu_{c 1,1}$ and $\nu_{c 2,1}$ are the change of the $\mathrm{Cl} \mathrm{NQR}$ frequency due to changes in its first neighbor, $\sigma^{2}=C_{c 1} C_{c 2} \sigma_{\mathrm{o}}^{2}, \bar{\nu}=C_{c 1 \nu 1}+C_{c 2 \nu 2}$, are the width and the mean frequency, respectively, that depend on the occupation factors and $\sigma_{\mathrm{o}}, \nu_{1}$, and $\nu_{2}$ the differences in NQR due to the two orientations of the molecules.

Because of the existence of two nonequivalent $\mathrm{Cl}$ atoms in the asymmetric unit, the NQR line must be fitted according to two distributions, as represented by eq 2 , one for each of the nonequivalent $\mathrm{Cl}$ atoms. According to the fractional occupancies of 60:40 and 80:20 for molecules 1 and 2, respectively, factoring parameters in eq 2 were settled as $C_{\mathrm{c} 1}=$ 0.6 and $C_{\mathrm{c} 2}=0.4$ and $C_{\mathrm{c} 1}=0.8$ and $C_{\mathrm{c} 2}=0.2$, for the $\mathrm{Cl}$ lines of molecules 1 and 2, respectively.

The excellent fit in Figure 5B shows that the split is similar for the two $\mathrm{Cl}$ atoms belonging to the inequivalent molecules in the cell, $\Delta \nu=\nu_{\mathrm{c} 1,1}-\nu_{\mathrm{c} 2,1}>160 \mathrm{kHz}$, and the broadening $\sigma$ is 40 and $60 \mathrm{kHz}$ for each contribution. These splitting and broadening values are similar to those found in orientationally disordered halobenzenes. ${ }^{43}$

According to the results from Fujimori and Asaji, ${ }^{35}$ at lowtemperature the ${ }^{35} \mathrm{Cl}$ spin-lattice relaxation time $T_{1}$ has a normal behavior due exclusively to torsional oscillations or lattice vibrations $\left(T_{1}\right)_{\text {lib, }}{ }^{46,47}$ while above $180 \mathrm{~K}$ another relaxation mechanism, following an Arrhenius behavior, appears. When molecular reorientations take place and quadrupole nucleus belongs to the reorienting group, $\left(T_{1}\right)_{\text {reo }}$ is proportional to the jump correlation time. ${ }^{48,49}$ When the nuclei do not jump but the electric field gradient (EFG) of the observed nuclei is modulated by reorientations of molecules or molecular groups a contribution, $\left(T_{1}\right)_{\mathrm{mod}}$, appears. ${ }^{47}$

Then, the temperature dependence of $T_{1}$ in molecular crystals can be usually expressed as the superposition of different contributions due to lattice vibrations $\left(T_{1}\right)_{\mathrm{ph}}$, slow reorientations of the quadrupolar atom, $\left(T_{1}\right)_{\text {reor, }}$ and reorientations of $\mathrm{Cl}$ neighbors groups, $\left(T_{1}\right)_{\bmod }$ :

$$
\begin{aligned}
\frac{1}{T_{1}} & =\left(\frac{1}{T_{1}}\right)_{\mathrm{ph}}+\left(\frac{1}{T_{1}}\right)_{\text {reor }}+\left(\frac{1}{T_{1}}\right)_{\mathrm{mod}}=A T^{\lambda}+\frac{k(\theta)}{\tau_{\text {reor }}} \\
& +\frac{1}{6}\left(\frac{q^{\prime}}{q}\right)^{2} \frac{\omega_{q}^{2} \tau_{\text {reo }}}{1+\omega_{q}^{2} \tau_{\text {mod }}^{2}}
\end{aligned}
$$

The exponent $\lambda$ for the lattice vibrational contribution is found to be between 2 and 3, but usually closer to $2 . \tau_{\text {reor }}$ is the jump correlation time, $k(\theta)$ is a factor that depends on the geometry of the reorientation and $\left(q^{\prime} / q\right)$ denotes the ratio between the fraction of the electric EFG affected by the molecular reorientation and the total EFG in the $\mathrm{Cl}$ site. For slow reorientations $\left(\omega_{\mathrm{Q}} \cdot \tau \gg 1\right)$ the modulation term is also proportional to $\tau_{\text {mod }}$ but because in molecular crystals $\left(q^{\prime} / q\right)<$ $0.05^{50}$ its contribution is smaller by a factor $6\left(q^{\prime} / q\right)^{-2} \approx 10^{4}$.

To have a reliable description of the disorder and the associated dynamics, we will use the information on dielectric results as well as the structural obtained details to reproduce the relaxation data in NQR.

In order to determine the molecular reorientational dynamics we subtracted the lattice vibrational contribution $\left(T_{1}\right)_{\mathrm{ph}}$, from the low-temperature range, to the $T_{1}$ experimental data. Then, within the slow reorientation regime we will have

$$
\frac{1}{T_{1}}-\left(\frac{1}{T_{1}}\right)_{\mathrm{ph}}=\Delta T_{1}^{-1}=\frac{k(\theta)}{\tau_{\text {reor }}}+\frac{1}{6}\left(\frac{q^{\prime}}{q}\right)^{2} \frac{1}{\tau_{\mathrm{mod}}}
$$

We use the dielectric relaxation times $\tau_{\alpha}$ and $\tau_{\beta}$ in eq 4 to reproduce $\Delta T_{1}^{-1}$ values matching $\tau_{\text {reor }}$ and $\tau_{\text {mod }}$ with some of the times $\tau_{\alpha}$ and $\tau_{\beta}$, respectively. Within this hypothesis, two consequences can be derived: (i) Because of the change of the neighbor molecular charges ( $S$ atom), there would be a contribution to the relaxation (modulation) which can be accounted with the factor $6\left(q^{\prime} / q\right)^{2}(<0.01)$. The only possibility that this process contributes to the observed values of $\Delta T_{1}^{-1}$ is that it occurs with the shortest relaxation time $\tau_{\beta}$ and with $\left(q^{\prime} / q\right)=0.03$. (ii) For reorientational jumps that involve the $\mathrm{Cl}$ atom, $k(\theta)=3 / 2\left(1-\cos ^{2}(\theta)\right)^{48}$ or $k(\theta)=(1-$ $\left.\left(3 \cos ^{2} \theta-1\right)^{2} / 4\right)^{49}$ depending on if the $\mathrm{Cl}$ atom jumps between two equivalent or nonequivalent potential wells with $\theta$ being the angle that the $\mathrm{C}-\mathrm{Cl}$ bond rotates. If $\mathrm{Cl}$ atom remains at the same position, there is no contribution $(\theta=0, k(\theta)=0)$. Although the X-ray structural solution does not support the inplane jump reorientation, we can try to see the contribution to $\mathrm{NQR}$ of this movement. In this case, $\theta$ will be $72^{\circ}$ giving a $k(\theta)$ factor of 1.5 , that would imply that the generated $\Delta T_{1}^{-1}$, with eq 4 , values will be below the experimental ones if we use $\tau_{\alpha}$ and even smaller with $\tau_{\beta}$. Therefore, this reorientation is clearly discarded. However, from the structural results, it is found that not only are the $\mathrm{S}$ atoms disordered but also the $\mathrm{Cl}$ with a small angle of rotation changing from $4^{\circ}$ at low temperature to ca. $10^{\circ}$ near the melting point. By assuming $\theta=10^{\circ}$ with $\tau_{\alpha}$, we have a value of $\Delta T_{1}^{-1}$ close to the experimental one.

In Figure $4 \mathrm{~B}$, the different relaxation times are shown: Long $\tau_{\alpha}$ and short $\tau_{\beta}$ relaxation times obtained from dielectric measurements, reorientational relaxation time $\Delta T_{1}^{-1}$ derived from $N Q R$ and $\Delta T_{1}^{-1}$ according to the equation

$$
\Delta T_{1}^{-1}=\frac{k\left(10^{\circ}\right)}{\tau_{\alpha}}+\frac{1}{6}(0.030)^{2} \frac{1}{\tau_{\beta}}
$$

that is, introducing the dielectric relaxation times in eq 4.

Thus, NQR data are compatible with two different movements conducting to the disorder determined from $\mathrm{X}$ ray diffraction and with the characteristic times found from dielectric spectroscopy: out-of-plane slow relaxations around the $\mathrm{C}-\mathrm{Cl} 2$-fold molecular axis and small angle fast reorientations of the $\mathrm{C}-\mathrm{Cl}$ bond.

The change of the $\mathrm{C}-\mathrm{Cl}$ bond jumps giving rise to the $\beta$ relaxation as demonstrated by the NQR analysis produces a noticeable change in the thermal expansion at around $185 \mathrm{~K}$, as 
revealed in Figure 3G (which is mainly due to the temperature variation of the lattice parameter $a$, see Figure 3A). Moreover, this change is accompanied by a change in the relaxation time distribution, as indicated in the temperature variation of the $c$ parameter of the CC equation (see inset in Figure 4A). This parameter decreases fast from high-temperature, close to the melting point, down to ca. $185 \mathrm{~K}$, reaching a value of 0.58 . The broadening of the $\beta$-relaxation peaks can be ascribed to the existence of heterogeneity of the dynamics of the system, which leads to a wider distribution of relaxation times. It gives rise also to a change in the dielectric strength of the $\beta$ relaxation (not shown). Such a widening (see inset in Figure $4 \mathrm{~A}$ ) is related to and goes with a slowing down of the thermal expansion of the lattice. It should be emphasized that as for the $\mathrm{C}-\mathrm{Cl}$ jumps originating the $\beta$-relaxation, the two molecules in the asymmetric unit do not contribute in the same way, as revealed by the angle of the jumps (see Figure $3 \mathrm{H}$ ). Whereas one molecule (molecule 2) changes the angular amplitude of the jumps at ca. $185 \mathrm{~K}$ on increasing temperature, molecule 1 keeps the slow increase with temperature without a noticeable change. This experimental fact contributes to prove the heterogeneity of this relaxation. As for the $c$ parameter of the $\alpha$-relaxation (out-of-plane molecular reorientations) as a function of temperature, it can be observed that does not feel the change in the lattice expansion, and thus the cooperativity of this process remains the same until the glass transition temperature $(164 \mathrm{~K})$.

In the light on these results, the specific heat anomaly reported by Fujimori and Oguni ${ }^{34}$ at $164 \mathrm{~K}$ is clearly associated with the freezing of the out-of-plane molecular reorientations, while the in-plane possible reorientations claimed from those authors are discarded. Consequently, the reported specific heat anomaly at $186 \mathrm{~K}^{34}$ cannot be originated by the in-plane reorientations. The authors fitted the relaxation enthalpy data around $186 \mathrm{~K}$ according to a stretched exponential and a value of $\beta^{\mathrm{KWW}}$ stretched exponent of 0.6 was obtained. They associated the low value of the exponent with a coupling between the two possible types of disorder, out-of-plane and in-plane, despite, as claimed from the authors, the glass transition temperature $(164 \mathrm{~K})$ for the slower process is relatively far away. According to our measurements, our $\beta^{\mathrm{KWW}}$ stretched exponent calculated ${ }^{51}$ through the $\beta^{\mathrm{KWW}}=$ $(c)^{1 / 1.23}$ is ca. 0.66 , so virtually the same. Nevertheless, the $\beta$ relaxation cannot be attributed to the in-plane reorientational process because it is incompatible with the structure, but as demonstrated by NQR analyses and coherently ascribed from the BDS experiments, to the $\mathrm{C}-\mathrm{Cl}$ jumps of one molecule of the asymmetric unit. Nevertheless, it should be noticed that inplane reorientational process can appear as short-lived molecular fluctuations (not detectable by X-ray diffraction) between one equilibrium site and a nonequilibrium site, with a short residence time, as it was found for some haloethane compounds. $^{25,26}$

\section{CONCLUSIONS}

We have investigated the dynamics and the structure of the crystalline phase of 2-Cl-thiophene by means of broadband dielectric spectroscopy and powder X-ray diffraction. The stable crystalline phase of this compound displays a complex triclinic $P 1$ lattice structure, with two molecules per asymmetric unit with site occupancies 80:20 and 60:40 for each molecule. The occupational disorder is associated with the existence of a reorientational dynamics consisting of out-of- plane $(\pi)$ rotations around roughly the $\mathrm{C}-\mathrm{Cl}$ bond, but such that the orientation of this bond is not the same before and after the out-of-plane reorientation. Such cooperative dynamics is responsible for the main dielectric loss (slow $\alpha$ relaxation), while the fast secondary $\beta$ relaxation is associated with small angle librations that change the position of the $\mathrm{Cl}$ atoms while leaving virtually invariant that of the $S$ atoms. The former relaxation is kinetically frozen $(\tau=100 \mathrm{~s})$ at $164 \mathrm{~K}$, which matches perfectly the glassy transition temperature obtained early from specific heat measurements. ${ }^{34}$ As for the thermal effect reported by the same authors at ca. $186 \mathrm{~K}$ and initially attributed to the onset of large-angle rotations around an axis perpendicular to the molecular plane (of $2 \pi / 5$ angular amplitude), which the authors of the previous studies referred to as in-plane rotations, lattice structure demonstrates that these rotations are discarded (at least between stable occupational sites). Moreover, it is demonstrated that such a thermal effect is related to a sudden increase of the amplitude of the $\mathrm{C}-\mathrm{Cl}$ angle between the two different occupational sites of one of the molecules in the asymmetric unit, an effect that is accompanied by a broadening in the relaxation time distribution of the fast $\beta$-relaxation.

The relaxation dynamics of the 2-Cl-thiophene molecule within the crystalline phase reported in the present study nicely explains the NQR measurements published in an earlier work. $^{35}$

As a general conclusion, this works demonstrates how "simple systems" displaying a disorder limited to a low number of degrees of freedom can be used to elucidate the microscopic origin of the $\alpha$-and $\beta$-relaxations.

\section{ASSOCIATED CONTENT}

\section{Supporting Information}

The Supporting Information is available free of charge on the ACS Publications website at DOI: 10.1021/acs.cgd.9b00871.

Figure S1: DSC measurement for the melting process. Table S1: Crystal structure information and Materials Studio results of the Rietveld refinement for the 2-Clthiophene (PDF)

\section{Accession Codes}

CCDC 1938423 contains the supplementary crystallographic data for this paper. These data can be obtained free of charge via www.ccdc.cam.ac.uk/data_request/cif, or by emailing data_request@ccdc.cam.ac.uk, or by contacting The Cambridge Crystallographic Data Centre, 12 Union Road, Cambridge CB2 1EZ, UK; fax: +44 1223336033.

\section{AUTHOR INFORMATION}

\section{Corresponding Author}

*Tel: +34 934016564. E-mail: josep.lluis.tamarit@upc.edu. ORCID

Michela Romanini: 0000-0002-1685-855X

Roberto Macovez: 0000-0001-5026-9372

Josep-Lluís Tamarit: 0000-0002-7965-0000

Notes

The authors declare no competing financial interest.

\section{ACKNOWLEDGMENTS}

This work has been partially supported by the Spanish Ministry of Economy and Competitiveness MINECO through Project FIS2017-82625-P, by the Generalitat de Catalunya under 
Project 2017SGR-42 and by CONICET, MinCyT Córdoba, and SECYT-UNC of Argentina.

\section{REFERENCES}

(1) Angell, C. A. Formation of Glasses from Liquids and Biopolymers. Science 1995, 267, 1924-1935.

(2) Angell, C. A.; Ngai, K. L.; McKenna, G. B.; McMillan, P. F.; Martin, S. W. Relaxation in Glassforming Liquids and Amorphous Solids. J. Appl. Phys. 2000, 88, 3113-3157.

(3) Ngai, K. L. Relaxation and Diffusion in Complex Systems, Partially Ordered Systems Series; Springer: New York, 2011.

(4) Suga, H.; Seki, S. Thermodynamic Investigation on Glassy States of PureSimple Compounds. J. Non-Cryst. Solids 1974, 16, 171-194.

(5) Adachi, K.; Suga, H.; Seki, S. Glassy Crystalline State-A nonEquilibrium State of Plastic Crystals. Bull. Chem. Soc. Jpn. 1970, 43, 1916.

(6) Brand, R.; Lunkenheimer, P.; Loidl, A. Relaxation Dynamics in Plastic Phases. J. Chem. Phys. 2002, 116, 10386-10401.

(7) Srinivasan, A.; Bermejo, F. J.; deAndres, A.; Dawidowski, J.; Zuñiga, J.; Criado, A. Evidence for a Supercooled Plastic-Crystal Phase in Solid Ethanol. Phys. Rev. B: Condens. Matter Mater. Phys. 1996, 53, 8172-8175.

(8) Vispa, A.; Romanini, M.; Ramos, M. A.; Pardo, L. C.; Bermejo, F. J.; Hassaine, M.; Krivchikov, A. I.; Taylor, J. W.; Tamarit, J. Ll. Thermodynamic and Kinetic Fragility of Freon113: the Most Fragile Plastic Crystal. Phys. Rev. Lett. 2017, 118, 105701.

(9) Gebbia, J. F.; Ramos, M. A.; Szewczyk, D.; Jezowski, A.; Krivchikov, A. I.; Horbatenko, Y. V.; Guidi, T.; Bermejo, F. J.; Tamarit, J. Ll. Glassy Anomalies in the Low-Temperature Thermal Properties of a Minimally Disordered Crystalline. Phys. Rev. Lett. 2017, 119, 215506.

(10) Romanini, M.; Barrio, M.; Capaccioli, S.; Macovez, M.; RuizMartin, M. D.; Tamarit, J. Ll. Double Primary Relaxation in a Highly Anisotropic Orientational Glass-Former with Low-Dimensional Disorder. J. Phys. Chem. C 2016, 120, 10614-10621.

(11) Romanini, M.; Negrier, Ph; Tamarit, J. Ll.; Capaccioli, S.; Barrio, M.; Pardo, L. C.; Mondieig, D. Emergence of Glass-like Dynamics in an Orientationally Ordered Phase. Phys. Rev. B: Condens. Matter Mater. Phys. 2012, 85, 134201.

(12) Moratalla, M.; Gebbia, J. F.; Ramos, M. A.; Pardo, L. C.; Mukhopadhyay, S.; Rudic, S.; Fernández-Alonso, F.; Bermejo, F. J.; Tamarit, J. Ll. Emergence of Glassy Features in Halomethane Crystals. Phys. Rev. B: Condens. Matter Mater. Phys. 2019, 99, 024301.

(13) Johari, G. P.; Goldstein, M. Viscous Liquids and the Glass Transition. II. Secondary Relaxations in Glasses of Rigid Molecules. J. Chem. Phys. 1970, 53, 2372.

(14) Zuriaga, M.; Pardo, L. C.; Lunkenheimer, P.; Tamarit, J. Ll.; Veglio, N.; Barrio, M.; Bermejo, F. J.; Loidl, A. A New Microscopic Mechanism for Secondary Relaxation in Glasses. Phys. Rev. Lett. 2009, 103, 075701.

(15) Martinez-Garcia, J. C.; Tamarit, J. Ll.; Pardo, L. C.; Barrio, M.; Rzoska, S. J.; Droz-Rzoska, A. Disentangling the Secondary Relaxations in the Orientationally Disordered Mixed Crystals: Cycloheptanol+Cyclooctanol Two-component System. J. Phys. Chem. B 2010, 114, 6099-6106.

(16) Ngai, K. L.; Lunkenheimer, P.; Leon, C.; Schneider, U.; Brand, R.; Loidl, A. Nature and Properties of the Johari-Goldstein $\beta$ Relaxation in the Equilibrium Liquid State of a Class of GlassFormers. J. Chem. Phys. 2001, 115, 1405-1413.

(17) Stevenson, J. D.; Wolynes, P. G. A Universal Origin for Secondary Relaxations in Supercooled Liquids and Structural Glasses. Nat. Phys. 2010, 6, 62-68.

(18) Kaminska, E.; Tarnacka, M.; Jurkiewicz, K.; Kaminski, K.; Paluch, M. High-Pressure Dielectric Studies on the Structural and Orientational Glass. J. Chem. Phys. 2016, 144, 054503.

(19) Tu, W.; Valenti, S.; Ngai, K. L.; Capaccioli, S.; Liu, D. Y.; Wang, L.-M. Direct Evidence of Relaxation Anisotropy Resolved by High Pressure in a Rigid and Planar Glass Former. J. Phys. Chem. Lett. 2017, 8, 4341-4346.
(20) Stillinger, F. H. A Topographic View of Supercooled Liquids and Glass Formation. Science 1995, 267, 1935-1939.

(21) Debenedetti, P. G.; Stillinger, F. H. Supercooled Liquids and the Glass Transition. Nature (London, U. K.) 2001, 410, 259.

(22) Ngai, K. L. Correlation between the secondary $\beta$-relaxation time at $\mathrm{Tg}$ with the Kohlrausch exponent of the primary $\alpha$ relaxation or the fragility of glass-forming materials. Phys. Rev. E: Stat. Phys., Plasmas, Fluids, Relat. Interdiscip. Top. 1998, 57, 7346.

(23) Ngai, K. L. An extended coupling model description of the evolution of dynamics with time in supercooled liquids and ionic conductors. J. Phys.: Condens. Matter 2003, 15, S1107-S1125.

(24) Ngai, K. L. Removal of Cooperativity in Glass-Forming Materials to Reveal the primitive $\alpha$-Relaxation of the Coupling Model. J. Phys.: Condens. Matter 1999, 11, A119-A130.

(25) Tripathi, P.; Mitsari, E.; Romanini, M.; Serra, P.; Tamarit, J. Ll.; Zuriaga, M.; Macovez, R. Orientational Relaxations in Solid (1,1,2,2)Tetrachloroethane. J. Chem. Phys. 2016, 144, 164505.

(26) Perez, S. C.; Zuriaga, M. J.; Serra, P.; Wolfenson, A.; Negrier, $\mathrm{Ph}$; Tamarit, J. Ll. Dynamic Characterization of Crystalline and Glass Phases of Deuterated 1,1,2,2 Tetrachloroethane. J. Chem. Phys. 2015, $143,134502$.

(27) Bohmer, R.; Loidl, A. Relaxation Dynamics in Molecular Alloys. 1. Annealed $\left(\mathrm{C}_{2} \mathrm{~F}_{6}\right)_{1-\mathrm{x}}\left(\mathrm{CClF}_{3}\right)_{\mathrm{x}}$ Mixtures. J. Chem. Phys. 1991, 94, 2143-2148.

(28) Ben Hassine, B.; Negrier, Ph; Romanini, M.; Barrio, M.; Macovez, R.; Kallel, A.; Mondieig, D.; Tamarit, J. Ll. Structure and Rreorientational Dynamics of 1-F-adamantane. Phys. Chem. Chem. Phys. 2016, 18, 10924-10930.

(29) Romanini, M.; Barrio, M.; Capaccioli, S.; Macovez, R.; RuizMartin, M. D.; Tamarit, J. Ll. DoublePrimaryRelaxation in a HighlyAnisotropicOrientationalGlass-Former withLow-Dimensional Disorder. J. Phys. Chem. C 2016, 120, 10614-10621.

(30) Damay, F.; Rodríguez-Carvajal, J.; André, D.; Dunstetter, F.; Szwarc, H. Orientational Ordering in the Low-Temperature Stable Phases of Deuterated Thiophene. Acta Crystallogr., Sect. B: Struct. Sci. 2008, 64, 589-595.

(31) Andre, D.; Figuiere, P.; Fourme, R.; Ghelfenstein, M.; Labarre, D.; Szwarc, H. Crystalline Thiophene-I Phase Diagram and Structures of Two Orientationally Disordered Crystalline Phases. Crystallographic Evidence for a Metastable Low Temperature Phase. J. Phys. Chem. Solids 1984, 45, 299-309.

(32) Figuiere, P.; Szwarc, H.; Oguni, M.; Suga, H. Calorimetric Study of Thiophene from 13 to $300 \mathrm{~K}$. Emergence of Two Glassy Crystalline States. J. Chem. Thermodyn. 1985, 17, 949-966.

(33) Krivchikov, A. I.; Stachowiak, P.; Pisarska, E.; Jezowski, A. Orientational Isotopic Effects in the Thermal Conductivity of $\mathrm{CH}_{4} /$ $\mathrm{CD}_{4}$ Solid Solutions. Low Temp. Phys. 2007, 33, 1061-1067.

(34) Fujimori, H.; Oguni, M. Thermodynamic Study of 2Chlorothiophene: Observation of Two Subsequent Glass Transitions in the Stable Crystalline State. J. Phys. Chem. Solids 1993, 54, 607612.

(35) Fujimori, H.; Asaji, T. ${ }^{35} \mathrm{CI} \mathrm{NQR}$ in Glassy Crystal of 2Chlorothiophene. Z. Naturforsch., A: Phys. Sci. 2000, 55, 183-185.

(36) Romanini, M.; Mitsari, E.; Tripathi, P.; Serra, P.; Zuriaga, M.; Tamarit, J. Ll.; Macovez, R. SimultaneousOrientationalandConformational Molecular Dynamics in Solid (1,1,2)-Trichloroethane. J. Phys. Chem. C 2018, 122, 5774-5783.

(37) M.S. Modeling, Materials Studio, version 5.5, https://www. 3 dsbiovia.com/products/collaborative-science/biovia-materialsstudio/.

(38) Harshbarger, W. R.; Bauer, S. H. An Electron Diffraction Study of the Structures of Thiophene, 2-Chlorothiophene and 2Bromothiophene. Acta Crystallogr., Sect. B: Struct. Crystallogr. Cryst. Chem. 1970, 26, 1010-1020.

(39) Dollase, W. A. Correction of Intensities for Preferred Orientation in Powder Diffractometry: Application of the March model. J. Appl. Crystallogr. 1986, 19, 267-272.

(40) Stoneham, A. M. Shapes of Inhomogeneously Broadened Resonance Lines in Solids. Rev. Mod. Phys. 1969, 41, 82-108. 
(41) Jung, J. K.; Seo, Y. M.; Choh, S. H. Characteristics of Impurity Effects in Isomorphous Crystals and Anisomorphous Mixtures Observed by ${ }^{14} \mathrm{~N}$ Nuclear Quadrupole Resonance. J. Chem. Phys. 1999, 110, 3913-3918.

(42) Cohen, M. H.; Reif, F. In Solid State Physics; Seitz, F.; Turnbull, D., Eds.; Academic Press: New York, 1957; Vol. 5, p 321.

(43) Pérez, S. C.; Zuriaga, M. A Model to Describe the Inhomogeneous Broadening of NQR Lines in Chlorohalobenzenes with Orientational or Substitutional Disorder. Solid State Nucl. Magn. Reson. 2017, 87, 18-23.

(44) Bussandri, P.; Zuriaga, M. J.; Martín, C. A. Room Temperature Nuclear Quadrupole Resonance Study of the Molecular Mixed Crystal p-Dichlorobenzene((1-x)) p-Dibromobenzene $((\mathrm{x}))$. J. Phys. Chem. Solids 1998, 59, 201-209.

(45) Perez, S. C.; Wolfenson, A.; Zuriaga, M. Isomorphism and Disorder in o-Chlorohalobenzenes Studied by NQR. Solid State Nucl. Magn. Reson. 2014, 57-58, 39-45.

(46) Jones, L. V.; Sabir, M.; Smith, J. A. S. ${ }^{35} \mathrm{Cl}$ Quadrupole SpinLattice Relaxation in p-Dichlorobenzene and 1,3,5-Trichlorobenzene Under Pressure. J. Phys. C: Solid State Phys. 1978, 11, 4077-4083.

(47) Woessner, D. E.; Gutowsky, H. S. Nuclear Pure Quadrupole Relaxation and its Temperature Dependence in Solids. J. Chem. Phys. 1963, 39, 440-456.

(48) Alexander, S.; Tzalmona, A. Relaxation by Slow Motional Processes. Effect of Molecular Rotations in Pure Quadrupole Resonance. Phys. Rev. 1965, 138, A845-A855.

(49) Gordeev, A. D.; Osipenko, A. N.; Soifer, G. B. NQR Data on the Activation Energy of Orientational Defects in Crystal Lattice. Crystallogr. Rep. 2001, 46, 1000-1004.

(50) Weiss, A. Crystal Field Effects in Nuclear Quadrupole Resonance, Nuclear Quadrupole Resonance. In Topics in Current Chemistry; Springer-Verlag: Berlin, 1972.

(51) Alvarez, F.; Alegra, A.; Colmenero, J. Relationship between the time-domain Kohlrausch-Williams-Watts and frequency-domain Havriliak-Negami relaxation functions. Phys. Rev. B: Condens. Matter Mater. Phys. 1991, 44, 7306-7312. 